\title{
DEVELOPMENT OF THE METHOD FOR ESTIMATING COMPLEX FORMATION USING THE ELECTROCHEMICAL IMPEDANCE SPECTROSCOPY ON THE EXAMPLE OF THE DOXYCYCLINE AND IRON (III) INTERACTION
}

\author{
Anna Dobrova, Yevhenii Antonenko, Olga Golovchenko, Nataliia Harna, \\ Svitlana Harna, Victoriya Georgiyants
}

\begin{abstract}
The aim. To develop an EIS method for study the interaction between medicinal products and metal salts on the example of the Doxycycline and iron (III) interaction.

Materials and methods. Measurements of the total impedance of the studied solutions have been performed using a vector circuit analyzer ZNB40 (Rohde \& Schwarz, Germany). The calculations of electrical models were performed using the software package EC-Lab V10.40. Measurement cell was made of Teflon, $1 \mathrm{ml}$ of volume, had 2 parallel nickel plated steel electrodes with diameter $6 \mathrm{~mm}$, distance between electrodes is $9 \mathrm{~mm}$. Basic electrical elements of model circuit were calculated according to type of electrochemical process that were described by Nyquist plot $\left(R_{\Omega}, R_{c t}, R_{S}\right.$, $C_{d}, C_{S}$ etc.). Solutions were prepared immediately before the measurement. Measurements were performed at a temperature of $296 \pm 3 \mathrm{~K}$. 6 control solutions of doxycycline and 6 control solutions of iron (III) chloride were prepared and measured. 11 study solutions at a molar ratio 1:6, 1:5, 1:4, 1:3, 1:2, 1:1, 2:1, 3:1, 4:1, 5:1, 6:1 were prepared and measured. Concentration of the solutions was $X \cdot 10^{-3} \mathrm{~mol} / \mathrm{L}$ respectively.

Results. EIS analysis of Nyquist curves of study solutions in the range of molar ratios 1: 6, 1: 5, 1: 4, 1: 3, 1: 2, 1: 1, 2: 1, 3: 1, 4: 1, 5: 1, 6: 1 showed a change in the dynamics of electrical resistance at a ratio of 1:1. In the aqueous solution at $T=296 \pm 3 \mathrm{~K}$ the constant formation of the solution of doxycycline hyclate and iron (III) chloride is 2.9. This value of the complexation constant indicates that doxycycline hyclate forms a stable metal-ligand complex with iron (III) ions. Conclusions. EIS method can be applied to study the interaction of medicinal products. Model of this study was created on the example of doxycycline hyclate and iron (III) chloride. Metal-ion complexation of these two molecules was once again confirmed by using the EIS method
\end{abstract}

Keywords: electrochemical impedance spectroscopy, complexation, antibiotics, drug interaction, doxycycline hyclate

(C) The Author(s) 2021

This is an open access article under the CC BY license (http://creativecommons.org/licenses/by/4.0).

\section{Introduction}

With the constant growth of the number of medicinal products on the market, the threat of polypharmacy is growing. Drug-drug interactions can lead to medicines ineffectiveness, cause adverse reactions or side effects $[1,2]$. According to the data obtained in the longitudinal studies the prevalence of clinically significant drug interactions is about $6 \%$ in patients taking $2-4$ drugs, $50 \%$ in those taking 5, and almost $100 \%$ in those taking 10 drugs $[1,2]$.

Therefore, the drug-drug interaction studies are highly important. They can be performed by both in vivo and in vitro studies. On the other hand, results of chemical, physicochemical experiments (in vitro) are important for estimating when in vivo studies may be necessary to fully define the drug interaction profile, and when they can be waived [3].

In vitro drug interactions studies can be performed by using different chemical and physicochemical methods. In our previous research we intended study the interaction between antibiotics and metal salts presented in the soft drinks and mineral water by UV-spectrophotometry and in vitro biowaiver test, but unfortunately these methods could not help us to establish the ratio of complexation $[4,5]$.
Electrochemical impedance spectroscopy (EIS) is an electrochemical technique that presents the signal as a function of frequency at a constant potential $[6,7]$. Although EIS is not a pharmacopoeial method, but it started to be used in the pharmaceutical and biomedical studies. New devices based on this method were created and used or rapid analysis of biological samples and optimization of many technological processes in the food and chemical industries [8]. Usage of EIS to study complexes of different transit metals like cobalt (II), nickel (II) and copper with several antibiotics and Schiff bases was reported $[9,10]$.

Tetracycline antibiotics are widely recognized as organic ligands that can interact with metal cations and form chelate complexes [11]. Although in the literature sources there is an information, that properties for complexation may differ within the group $[12,13]$.

Considering mentioned above for the study it was decided to use doxycycline as a study object to model the interaction of doxycycline (Fig. 1) and the iron (III) in the aqueous solution and to study it by using the EIS method. Consequently, the results of the doxycycline and $\mathrm{Fe}^{3+}$ interaction study obtained by EIS and electrochemical modeling can be compared with estimates reported 
before and provide an appropriate chemical interpretation of the processes that take place.<smiles>CC1C(O)=C(C(N)=O)C(=O)C2(O)C(O)=C3C(=O)c4c(O)cccc4C(C)C3C(N(C)C)C12</smiles>

Fig. 1. Doxycycline hyclate

The aim of the research. To substantiate and develop approaches to the study of metals and doxycycline complexation using the EIS method. To create a model (tool) for studying the interaction by using this method, for its further use to study the interaction of other medicinal products.

\section{Planning (methodology) of research}

At the first stage the total impedance of six iron (III) chloride solutions, the concentration of which varied from $1 \cdot 10^{-3}$ to $6 \cdot 10^{-3} \mathrm{~mol} / \mathrm{L}$, was measured using EIS. The total impedance of six solutions of doxycycline hyclate, the concentration of which varied from $1 \cdot 10^{-3}$ to $6 \cdot 10^{-3} \mathrm{~mole} / \mathrm{L}$.

At the second stage, 11 aqueous solutions of doxycycline hyclate and iron (III) chloride were prepared in the following molar ratios: 1:6, 1:5, 1:4, 1:3, 1:2, 1:1, $2: 1,3: 1,4: 1,5: 1,6: 1$, and for each of them the electrochemical impedance was measured and the electroimpedance spectral characteristics were constructed in the form of Nyquist plot.

For six molar concentrations of doxycycline and iron (III) at the range $1 \cdot 10^{-3}-6 \cdot 10^{-3} \mathrm{~mol} / \mathrm{L}$, as well as for such ratios of concentrations of Dox and the iron (III) as $1: 6,1: 5,1: 4,1: 3,1: 2,1: 1,2: 1,3: 1,4: 1,5: 1,6: 1$ the frequency dependences of the real and imaginary parts of the impedance on which the Nyquist curves were constructed. Thereafter, a qualitative analysis of the Nyquist curves was performed, equivalent electrical models were constructed and their electrical elements $\left(\mathrm{R}_{\Omega}, \mathrm{C}_{\mathrm{d}}, \mathrm{Z}_{\mathrm{f}}\right)$ were calculated.

\section{Materials and methods}

\section{1. Reagents}

Doxycycline hyclate substance used for preparation of the study solutions was purchased from Yangzhou Liberty Pharmaceutical Co., Ltd, China. Iron (III) chloride hexahydrate substance was from Sigma Aldrich, Germany. Grade "A" glassware were used during the study. All used reagents were of analytical grade. Purified water was used as a main solvent [14].

\section{2. Preparation of samples}

The following aqueous solutions were prepared (control solutions): doxycycline hyclate 1, 2, 3, 4, 5, 6; iron (III) chloride 1, 2, 3, 4, 5, 6 (with concentration respectively $\mathrm{X} \cdot 10^{-3} \mathrm{~mol} / \mathrm{L}$ ). Stock aqueous solutions of doxycycline and iron (III) chloride were prepared. Study solutions were obtained from stock to receive mixed solution of doxycycline and $\mathrm{Fe}^{3+}$ in the following molar ratio: 1:6, 1:5, 1:4, 1:3, 1:2, 1:1, 2:1, 3:1, 4:1, 5:1, 6:1 (with concentration respectively $\mathrm{X} \cdot 10^{-3} \mathrm{~mol} / \mathrm{L}$ ). Solutions were prepared immediately before the measurement. Measurements were performed at a temperature of $296 \pm 3 \mathrm{~K}$.

\section{3. Instrumentation and method}

The measuring cell, which includes electrodes, and the electrochemical processes occurring in it during measurement, can be modeled by an equivalent electrical circuit [15]. In the electrochemical cell when measuring the total impedance of the studied chemical solutions, there is a charge transfer between them and the electrodes. Thereby there are certain Faraday processes, thus the most appropriate electrical model is the equivalent Randles electrical circuit $[6,15]$. Basic electrical elements of model circuit were calculated according to type of electrochemical process that were described by Nyquist plot $\left(\mathrm{R}_{\Omega}, \mathrm{R}_{\mathrm{ct}}, \mathrm{R}_{\mathrm{S}}, \mathrm{C}_{\mathrm{d}}, \mathrm{C}_{\mathrm{S}}\right.$ etc.) $[6,15]$. Constant phase elements together with Warburg resistance characterize the features and dynamics of chemical processes in the studied solutions [16]. Thus, by building the appropriate electrical model based on the results of total impedance measurements and calculating its elements, it is possible to assess the peculiarities of chemical processes and draw conclusions about the complexation between the studied substances and metal salts.

During our research, both approaches were used qualitative estimation by Nyquist curves and modeling by values of an electric circuit, as well as quantitative estimation of its elements and determination by their dynamics of electrochemical processes occurring in the studied solutions.

Measurements of the total impedance of the investigated solutions were performed using a vector circuit analyzer manufactured by Rohde \& Schwarz ZNB40.

Measurement cell used in the study was made of Teflon, $1 \mathrm{ml}$ of volume, had 2 parallel nickel plated steel electrodes with diameter $6 \mathrm{~mm}$, distance between electrodes is $9 \mathrm{~mm}$.

The calculation of electrical models was performed using the software package EC-Lab V10.40, which implemented randomization and simplex methods for calculating the parameters of electrical circuits for a full cycle of iterations to achieve 5000 iterations.

Given that solutions of substances in water were studied, the frequency ranges in which the electrochemical impedance was measured ranged from $100 \mathrm{kHz}$ to $1 \mathrm{GHz}$. Both the frequency dependences of the impedance change and its dependence on the ratio of the concentrations of the studied objects were determined. The measurement of the total impedance consisted of determining the frequency dependence of its real $\left(\mathrm{Z}_{\mathrm{Re}}\right)$ and imaginary parts $\left(Z_{\text {Im }}\right)$. Further such functional dependencies as the dependence of the total impedance modulus on the frequency $-|\mathrm{Z}|=\mathrm{Z}(\mathrm{f})$; the dependence of the phase angle of the total impedance on the frequency $-\phi=\Phi(f)$ and the relationship between the real and imaginary parts of the total impedance in the form of the dependence: $\mathrm{Z}_{\mathrm{Im}}=\zeta\left(\mathrm{Z}_{\mathrm{Re}}\right)$ were determined [15]. 


\section{Result}

Fig. 2 shows the electroimpedance spectral characteristics of solutions of different concentrations of iron (III) chloride and doxycycline hyclate in the form of Nyquist curves. The diagrams in Fig. 2a show that for all concentrations of iron (III) chloride solutions the dependence has the same shape in the form of one semicircle, which begins at higher frequencies and does not have a clearly defined tail on the low frequency side. This indicates that the kinetic processes of charge transfer predominate in the chemical system, while mass transfer due to diffusion processes is almost absent. As the concentration of iron (III) chloride increases, the size of the semicircle of the Nyquist curves decreases.

At the same time, its shape does not change and the size of the tail, which is characterized by the Warburg impedance, does not increase, and the transition between it and the semicircle remains clear.
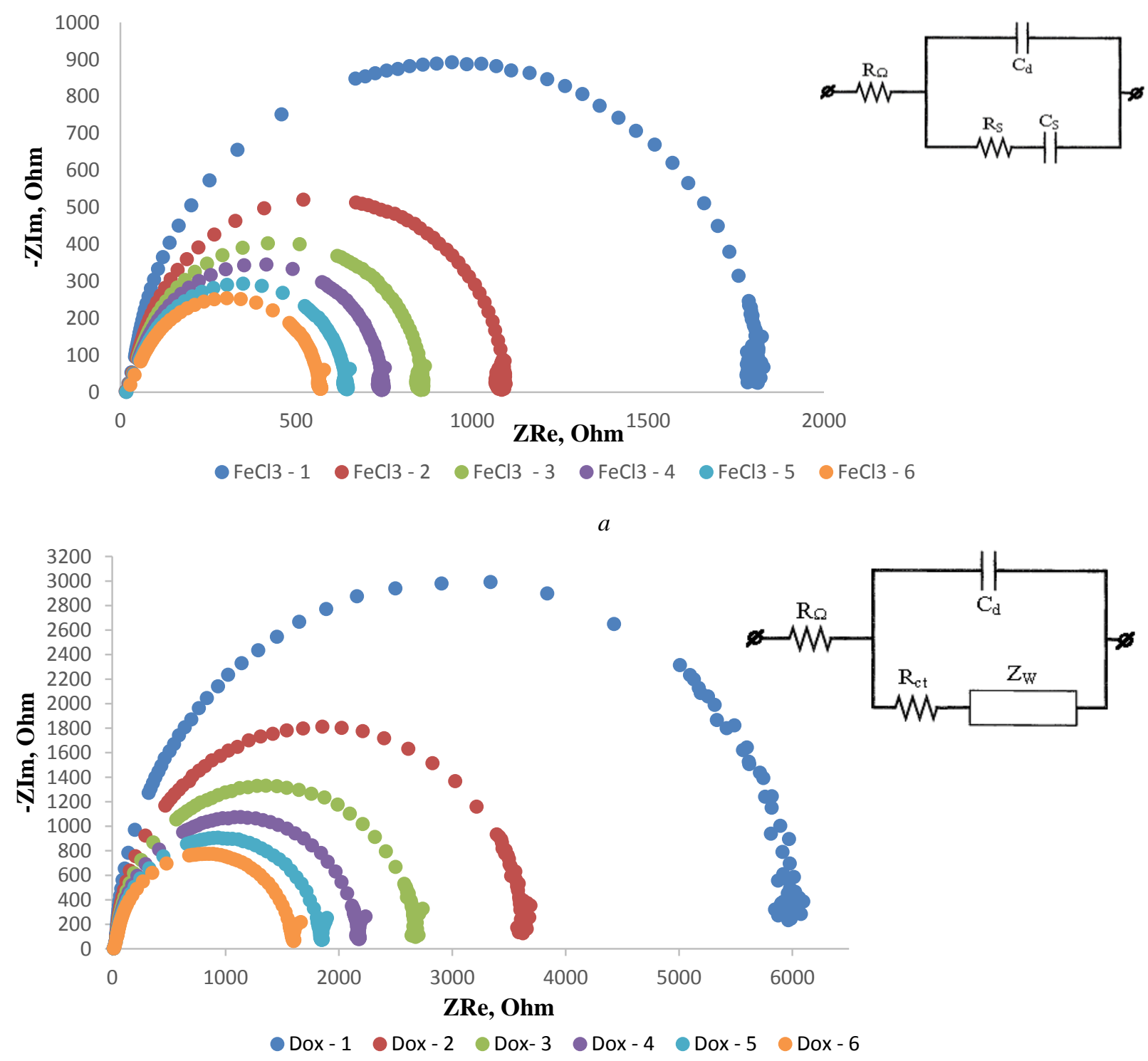

$b$

Fig. 2. Nyquist curves with inset of equivalent electrical circuit of the measuring cell for 6 molar concentrations of solutions of $a-\mathrm{FeCl}_{3} ; b-$ doxycycline hyclate

As shown by the first stage of the experiment, an increase in the concentration of iron (III) chloride leads to a decrease in the resistance of the electrochemical system and, accordingly, to an increase in conductivity due to an increase in iron ions in solution. At the same time, the decrease in the concentration of iron (III) chloride does not lead to the replacement of the dominant kinetic processes of charge transfer by mass transfer processes. In general, we can conclude that in an electro- chemical cell with a solution of iron (III) chloride Faraday processes take place. To model them, an equivalent electrochemical circuit with a series connection of a resistor $\mathrm{R}_{\Omega}$ (which simulates the resistance of the solution in the measuring cell), and a parallel connection created by the measuring electrodes of the capacitance $C_{d}$ with electrical resistance of $R_{S}$ and pseudocapacitance $C_{S}$ (which determine electrochemical processes occurring in the studied solutions can be chosen) (Fig. 2, a). 
The values of calculated equivalent electrical elements for the electrochemical impedance model of $\mathrm{FeCl}_{3}$ solution are given in Table 1 .

They confirm that with increasing concentration of iron (III) chloride there is a decrease in the value of the electrical resistance of charge transfer $\mathrm{R}_{\mathrm{S}}$ three times, from $1763 \mathrm{Ohms}\left(\mathrm{C}_{1}=1 \cdot 10^{-3} \mathrm{~mol} / \mathrm{L}\right)$ to $524 \mathrm{Ohms}$ $\left(\mathrm{C}_{6}=6 \cdot 10^{-3} \mathrm{~mol} / \mathrm{L}\right)$.
The value of the pseudocapacitance of $\mathrm{C}_{\mathrm{S}}$ is doubled from $25.65 \mathrm{pF}$ to $56.91 \mathrm{pF}$ with increasing concentration of iron (III) chloride from $1 \cdot 10^{-3} \mathrm{~mole} / \mathrm{L}$ to $6 \cdot 10^{-3} \mathrm{~mole} / \mathrm{L}$. Thus, the total conductivity of the electrochemical system of $\mathrm{FeCl}_{3}$ solution increases with increasing concentration, because of the dominance of the charge transfer process due to the increase in the concentration of $\mathrm{Fe}^{3+}$ and $\mathrm{Cl}^{-}$ions.

Table 1

Parameters of electrochemical impedance for the iron (III) and doxycycline hyclate solutions of different concentrations

\begin{tabular}{|c|c|c|c|c|c|c|c|}
\hline $\begin{array}{c}\text { Concentra- } \\
\text { tion of the } \\
\text { solution, } \\
10^{-3} \text { mole/L }\end{array}$ & $\mathrm{R}_{\Omega}, \mathrm{Ohm}$ & $\mathrm{C}_{\mathrm{d}}, 10^{-12} \mathrm{~F}$ & $\mathrm{R}_{\mathrm{S}}, 10^{3} \mathrm{Ohm}$ & $\begin{array}{c}\mathrm{C}_{\mathrm{S}}, \\
10^{-9} \mathrm{~F}\end{array}$ & $\begin{array}{c}\mathrm{Q}_{2}, \\
10^{-9} \mathrm{~F}_{\mathrm{s}}{ }_{2}{ }^{-1)}\end{array}$ & $\mathrm{a}_{2}$ & $\sigma_{2}$ \\
\hline \multicolumn{7}{|c|}{ Iron (III) chloride solution } \\
\hline 1
\end{tabular}

For a solution of doxycycline hyclate, the spectral curves of Nyquist have the shape of a semicircle, the size of which decreases with increasing concentration of the substance (Fig. 2, b). It means that kinetic charge transfer processes prevails in this chemical system, as well as for a solution of iron (III) chloride. However, in contrast to iron (III) chloride, the electrochemical impedance curves for doxycycline hyclate have a more pronounced Warburg impedance, which increases with increasing concentration of the substance (Fig. 2, b). The appearance of the Warburg impedance in this electrochemical system can be explained by the fact that the molecule of doxycycline hyclate has a more complex structure. In a medium of purified water ( $\mathrm{pH} 5.5-7.0$ ), i.e. at neutral $\mathrm{pH}$, the molecule of doxycycline has structure of zwitterion [13]. The dimethylamino group is responsible for the basic properties, while the acidic properties are manifested due to phenolic hydroxyl in the ring D and enol groups. This structure of the molecule explains the fact that when measuring the electrochemical impedance at low frequencies under the action of an electric field, the diffusion of matter occurs due to the processes of electrooxidation and electroreduction. This leads to the appearance of the Warburg impedance on the spectral curves of Nyquist (Fig. 2, b). Considering this, to calculate the electrochemical impedance system of the doxycycline hyclate solution, we used an equivalent electrical circuit $\mathrm{R}_{\Omega}+\mathrm{C}_{\mathrm{d}} /\left(\mathrm{R}_{\mathrm{ct}}+\mathrm{C}_{3} /\left(\mathrm{R}_{3}+\mathrm{W}_{3}\right)\right.$ ) (Fig. 3, b). In the Table 1 the values of equivalent electrical elements for the above electrochemical model of the impedance of a solution of doxycycline hyclate are given.

With increasing concentration of doxycycline hyclate there is a decrease in the value of electrical resistance $\mathrm{R}_{\mathrm{S}}$ in four times, from $5966 \mathrm{Ohm}\left(\mathrm{C}_{1}=1 \cdot 10^{-3} \mathrm{~mole} / \mathrm{L}\right)$ to $1554 \mathrm{Ohm}\left(\mathrm{C}_{6}=6 \cdot 10^{-3} \mathrm{~mole} / \mathrm{L}\right)$, while significantly increasing the mass transfer coefficient $\left(\sigma_{2}\right)$, which characterizes the sum of process contributions oxidation and reduction in this electrochemical system.

The dynamics of the constant phase element $-\mathrm{Q}_{2}$ and $\mathrm{a}_{2}$ - the deviation of the model parameters characterizes that when the concentration of doxycycline hyclate increases, the parameters of the electric model deviate from the pseudocapacitance and the influence of the Warburg impedance increases (Table 1). This can be explained by the fact that water molecules are partially replaced by zwitterionic ions of doxycycline, the number of these ions increases and the total conductivity of the solution of doxycycline hyclate. Moreover, the $\mathrm{pH}$ of the doxycycline hyclate solution decreases, so the number of negatively charged ions increases due to phenolic hydroxyl in the ring D and enol groups, and due to their mass transfer increases.

As it can be seen from the Nyquist diagrams (Fig. 3), the addition of iron (III) chloride to doxycycline hyclate lead to a change in electroimpedance spectral characteristics either in shape (the diameter of the impedance semicircle decreased compared to the corresponding diagrams for pure doxycycline hyclate solution (Fig. 2, b), or the clear appearance of the Warburg im- 
pedance. This occurs even at the lowest concentration of doxycycline hyclate $-1 \cdot 10^{-3}$ mole/L.

It is also important to note that as the concentration of iron (III) chloride increases, the diameter of the electroimpedance semicircle decreases (Fig. 3,a). This qualitatively indicates a decrease in the value of the electrical resistance $\mathrm{R}_{\mathrm{S}}$ of this electrochemical system and, accordingly, an increase in the electrochemical conductivity with increasing concentration of iron (III) chloride. The maximum electrical resistance is observed at a ratio of concentrations of solutions of doxycycline hyclate and iron (III) chloride $-1: 1$, and the minimum at 1:6. The study of solutions for which the concentration of doxycycline hyclate increased showed that with increasing concentration of doxycycline hyclate relative to iron (III) chloride there is also a decrease in the electrical resistance $\mathrm{R}_{\mathrm{S}}$ of this electrochemical system and, accordingly, an increase in conductivity (Fig. 3b).

The maximum electrical resistance is observed at a ratio of concentrations of solutions of doxycycline hyclate and iron (III) chloride $-1: 1$, and the minimum at $6: 1$.
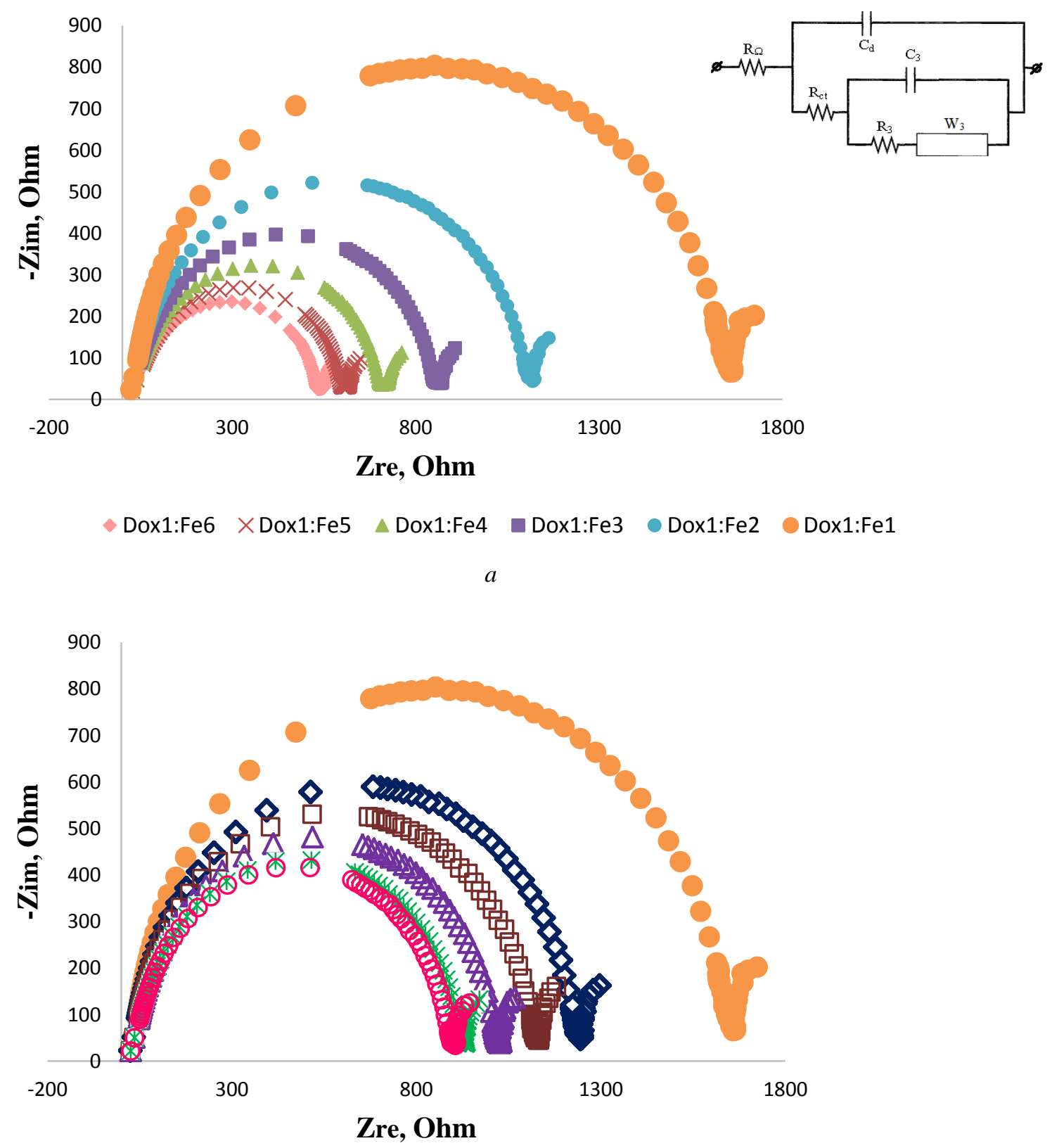

Dox1:Fe1 $\diamond$ Dox2:Fe1 $\square$ Dox3:Fe1 $\triangle$ Dox4:Fe1 $*$ Dox5:Fe1 ODox6:Fe1

$b$

Fig. 3. Nyquist curves for doxycycline and iron (III) chloride solutions in different concentration ratios, measured at a temperature of $295 \mathrm{~K}: a$ - increase in $\mathrm{Fe}^{3+}$ concentration; $b$ - increase in doxycycline concentration 
The model parameters of the electrochemical system of aqueous solutions of doxycycline hyclate and iron (III) chloride in molar ratios of concentrations: 1:6, 1:5, 1:4, 1:3, 1:2, 1:1, 2:1, 3:1, 4:1, 5:1, 6:1 are given in Table 2.

Analysis of the dynamics of EIS parameters showed that when the ratio of the concentration of $\mathrm{Fe}^{3+}$ ions to the amount of doxycycline in the studied solutions there is a slight fluctuation of the pseudocapacitance of the measuring cell $\mathrm{C}_{\mathrm{d}}$ in the range of $4.9 \ldots 5.6 \mathrm{pF}$ and electrical resistance of the test solution $\mathrm{R}_{\Omega}$ in the range of $39.33 \ldots 31.36$ Ohms. This variability can be ignored, and these indicators can be considered almost unchanged. Moreover, the EIS parameters that characterize the redox reactions and other electrochemical processes occurring in the investigated solutions significantly depend on the ratio of molar concentrations. Thus, when the concentration of $\mathrm{Fe}^{3+}$ ions decreases from $6 \cdot 10^{-3} \mathrm{moles} / \mathrm{L}$ to $1 \cdot 10^{-3} \mathrm{moles} / \mathrm{L}$, there is an increase in the charge transfer resistance $\mathrm{R}_{\mathrm{ct}}$ four times, which reaches a maximum value of $1.606 \mathrm{kOhm}$ at a ratio of $1: 1$ (Table 2). Then there is a gradual decrease of almost two times with increasing the concentration of doxycycline from $1 \cdot 10^{-3} \mathrm{moles} / \mathrm{L}$ to $6 \cdot 10^{-3} \mathrm{moles} / \mathrm{L}$ (Table 2 ). Herewith, the inverse dynamics is observed for the pseudocapacitance $\mathrm{Q}_{2}$, which characterizes the processes of mass transfer and diffusion. Thus, reducing the molar concentration of $\mathrm{Fe}^{3+}$ ions from $6 \cdot 10^{-3}$ moles $/ \mathrm{L}$ to $1 \cdot 10^{-3}$ moles/L leads to a decrease in pseudocapacitance by 17 times from $1301 \mathrm{pF}$ to $75.16 \mathrm{pF}$. A further increase in the concentration of doxycycline hyclate leads to a decrease in the value of the pseudocapacitance of $\mathrm{Q}_{2}$ by half (Table 2). Thus, at a ratio of molar concentrations of doxycycline hyclate and iron (III) chloride 1:1 for both the resistance of charge transfer $R_{S}$ and for the pseudocapacitance $\mathrm{Q}_{2}$, a change in the dynamics is observed, which is a confirmation of the complexation process at this point.

To further quantify the stability of complexation according to the indicators of electrochemical resistance of charge transfer (Tab. 2), the molar conductivities of the investigated solutions were calculated, the dependence of which on the ratio of molar concentrations [Dox]: $\left[\mathrm{Fe}^{3+}\right]$ are given in Fig. 4 .

Table 2

Parameters of electrochemical impedance of solutions of doxycycline hydrochloride and $\mathrm{FeCl}_{3}$ in different ratios of molar concentrations [Dox]:[ $\left.\mathrm{Fe}^{3+}\right]$

\begin{tabular}{|c|c|c|c|c|c|c|c|c|c|c|c|}
\hline$[\mathrm{Dox}]:\left[\mathrm{Fe}^{3+}\right]$ & $\mathrm{R}_{\Omega}, \mathrm{Ohm}$ & $\begin{array}{c}\mathrm{Q} 1, \\
\left.10^{-12} \mathrm{~F}^{\left(\mathrm{a}^{-1}\right)}{ }_{1}\right)\end{array}$ & $a_{1}$ & $\begin{array}{c}\mathrm{C}_{\mathrm{d}}, \\
10^{-12} \mathrm{~F}\end{array}$ & $\begin{array}{c}\mathrm{R}_{\mathrm{ct}} \\
10^{3} \mathrm{Ohm}\end{array}$ & $\begin{array}{c}\sigma_{2}, \\
\mathrm{Ohm} \cdot \mathrm{s}^{-1 / 1}\end{array}$ & $\begin{array}{c}\mathrm{Q} 2, \\
10^{-9} \mathrm{~F}^{\left(\mathrm{a}^{-}-1\right)}\end{array}$ & $a_{2}$ & $\begin{array}{c}\mathrm{C}_{3}, \\
10^{-9} \mathrm{~F}\end{array}$ & $\begin{array}{l}\mathrm{R}_{3}, \\
\text { Ohm }\end{array}$ & $\begin{array}{c}\sigma_{3}, \\
\mathrm{Ohm} \cdot \mathrm{s}^{-1 / 2}\end{array}$ \\
\hline $1: 6$ & 39.33 & 5.698 & 1 & 5.698 & 0.486 & 45925 & 1301.00 & 0.680 & 6.542 & 10.7 & 60563 \\
\hline $1: 5$ & 35.11 & 5.158 & 1 & 5.158 & 0.550 & 49898 & 1061.00 & 0.689 & 6.317 & 19.7 & 68920 \\
\hline $1: 4$ & 36.80 & 5.382 & 1 & 5.382 & 0.659 & 57248 & 987.70 & 0.684 & 5.66 & 22.4 & 74358 \\
\hline $1: 3$ & 35.20 & 5.235 & 1 & 5.235 & 0.809 & 57760 & 504.60 & 0.732 & 6.87 & 29.5 & 85653 \\
\hline $1: 2$ & 33.41 & 5.074 & 1 & 5.074 & 1.064 & 58678 & 169.40 & 0.807 & 8.654 & 78.4 & 112917 \\
\hline $1: 1$ & 31.36 & 4.912 & 1 & 4.912 & 1.606 & 75640 & 75.16 & 0.845 & 8.182 & 450.4 & 5524 \\
\hline $2: 1$ & 32.79 & 5.016 & 1 & 5.016 & 1.196 & 61743 & 71.64 & 0.863 & 13.45 & 378.1 & 13.94 \\
\hline $3: 1$ & 33.28 & 5.079 & 1 & 5.079 & 1.081 & 61545 & 94.75 & 0.844 & 10.23 & 350.9 & 18.89 \\
\hline $4: 1$ & 33.91 & 5.131 & 1 & 5.131 & 0.977 & 55162 & 98.67 & 0.839 & 11.44 & 329.0 & 21.84 \\
\hline $5: 1$ & 34.65 & 5.170 & 1 & 5.170 & 0.882 & 53196 & 141.60 & 0.826 & 12.00 & 292.3 & 38.33 \\
\hline $6: 1$ & 34.90 & 5.196 & 1 & 5.196 & 0.854 & 50668 & 161.50 & 0.821 & 12.65 & 274.7 & 108.90 \\
\hline
\end{tabular}

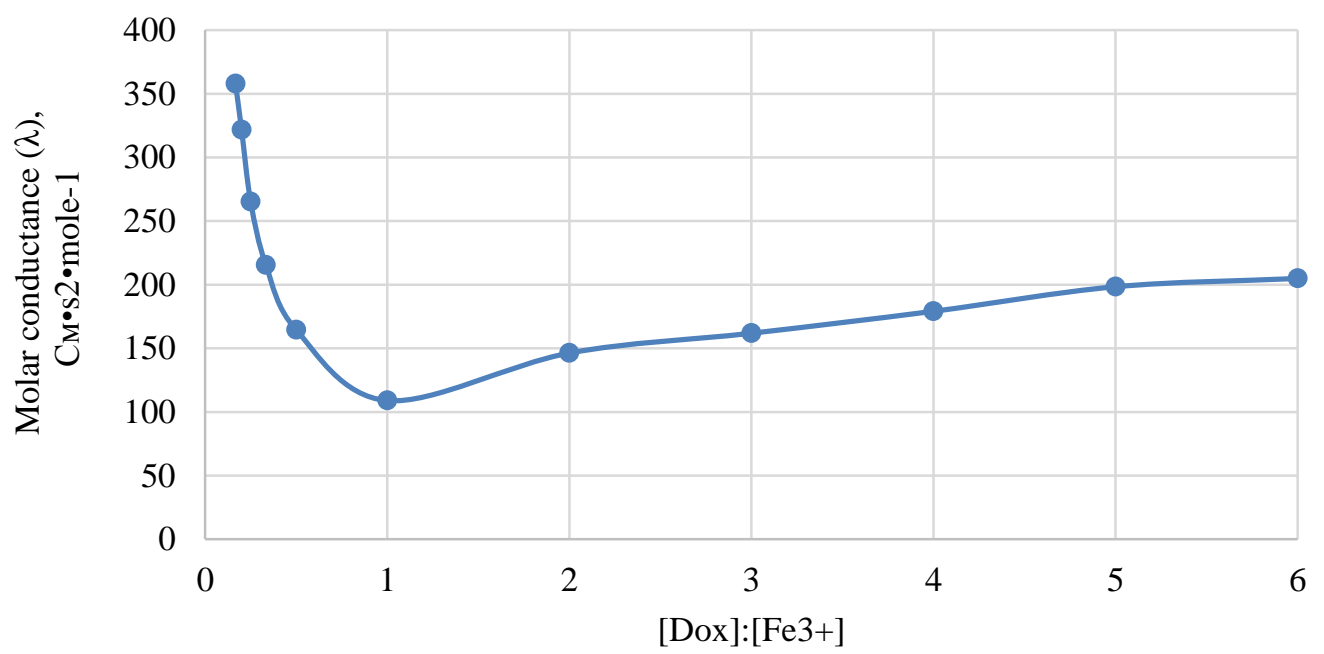

Fig. 4. Dependence of molar conductivity on the ratio of molar concentrations [Dox]: $\left[\mathrm{Fe}^{3+}\right]$ dissolved in water at a temperature of $295 \mathrm{~K}$

As can be seen from Fig. 4, there is a constant decrease in the molar conductivity of solutions for $\mathrm{Fe}^{3+}$ cations. This clearly indicates that the complexes formed were less mobile than the free solvated cations. Further, 
the slope of the molar conductivity curve changes at the point where the molar ratio of doxycycline hyclate and $\mathrm{Fe}^{3+}$ concentrations is $1: 1$, which indicates the formation of complexes at this point.

The stability constant (complexation) was calculated by the formula (1) [17]:

$$
K_{M L+}=\frac{a_{1} a_{2}}{a_{2} a_{3}[L]_{t}-a_{1} a_{3}[M]_{t}},
$$

where $a_{1}=\lambda_{M+}-\lambda, a_{2}=\lambda_{M+}-\lambda_{M L}, a_{3}=\lambda-\lambda_{M L}$,

$$
\lambda=\frac{1000 \cdot k}{C},
$$

$\lambda_{M+}, \quad \lambda_{M L}-$ molar conductivities of the metal salt solution, as well as the test solution of the antibacterial drug and the corresponding metal salt, $[L]_{t}$ - molar concentration of ligand (doxycycline), $[M]_{t}$ - molar concentration of cation (metal salt).

Taking into account the previously calculated molar conductivities of the investigated solutions, it was determined that at $\mathrm{T}=296 \pm 3 \mathrm{~K}$ and in purified water the constant formation of the solution of doxycycline hyclate and iron (III) chloride is $\log _{10}\left(\mathrm{~K}_{\mathrm{ML}+}\right)=2.9$ units. This value of the complexation constant indicates that doxycycline hyclate forms a stable metal-ligand complex with iron (III) ions.

\section{Discussion}

EIS analysis of Nyquist curves of doxycycline hyclate and iron (III) chloride aqueous solutions in the range of molar ratios 1:6, 1:5, 1:4, 1:3, 1:2, 1:1, 2:1, 3:1, $4: 1,5: 1,6: 1$ showed a change in the dynamics of electrical resistance at a ratio of $1: 1$, which can be considered a qualitative sign of complexation at this point.

It is also important to note that the charge transfer resistance of the studied electrochemical systems significantly exceeds the value of the Warburg impedance $\mathrm{R}_{\mathrm{S}}>\mathrm{R}_{3}$ (Table 2). This means that the activity of iron (III) ion transfer is higher than the activity of the diffusion transfer process in the oxidation of doxycycline hyclate, despite the fact that with decreasing concentration of iron (III) ions, the mass transfer coefficients $\sigma_{2} \mathrm{i}$ $\sigma_{3}$ increase. At the same time, both for the previous electrochemical parameters and for the dynamics of mass transfer coefficients $\sigma_{2} \mathrm{i} \sigma_{3}$, the ratio [Dox]:[Fe $\left.{ }^{3+}\right]=1: 1$ is the transition point of dynamics from decrease to increase.

In general, the study confirmed the complexation of iron(III) and doxycycline as it is reported in the results of previous studies conducted using other physicochemical methods [18, 19]. Khammas et al. studied and reported the formation of iron (III)-doxycycline metal complexes in acidic medium by UV-spectrophotometry; by using Job's method they have estimated the ratio of $\left[\mathrm{Fe}^{3+}\right]:[\mathrm{Dox}]$ as 1:2 [18]. Javed and Zahir also reported the presence of complexation at the metal-ligand molar ratio 1:2 estimated by UV-spectrophotometry and thermodynamic studies. The variation between the results can be explained by the differences of the methodology approaches. The first and foremost is the different $\mathrm{pH}$ of the studies, as in our research the neutral solvent was used, and the acidic one was used in the studies referred above.

Research limitations. The choice of dissolution medium, study cell and electrode material can influence the electrochemical process significantly, hence should be carefully considered.

Prospects for further research. It is planned to further conduct a study of the complexation of doxycycline by the EIS method with other metal salts that may interact. Moreover, to expand research to study the interaction of other antibiotics, using the developed model.

\section{Conclusions}

Conducted study of the possibility of using the EIS method as a tool for assessing the complexation of antibacterial drugs and metal salts on the example of the interaction of doxycycline hyclate and salts of iron (III). Possibility of doxycycline and iron (III) complexation was confirmed both qualitatively and quantitatively. According to the change in the slope of the molar conductivity curve, as well as the maximum value of electrical resistance, the ratio at which the complexes are formed is $1: 1$. The constant of the complex formation was calculated using estimated molar conductivities values is 2.9. The obtained results showed the effectiveness and possibility of using the EIS to further study the chemical interactions of drugs and metal salts.

\section{Conflict of interest}

The authors declare that they have no conflicts of interest.

\section{References}

1. Rana, D., Suthar, J., Malhotra, S., Patel, V., Patel, P. (2014). A study of potential adverse drug-drug interactions among prescribed drugs in medicine outpatient department of a tertiary care teaching hospital. Journal of Basic and Clinical Pharmacy, 5 (2), 44-48. doi: http://doi.org/10.4103/0976-0105.134983

2. Selvarajan, S., Das, S., Behera, S., Xavier, A., Dharanipragada, S. (2019). Are drug-drug interactions a real clinical concern? Perspectives in Clinical Research, 10 (2), 62-66. doi: http://doi.org/10.4103/picr.picr_55_18

3. Robertson, S., Penzak, S. R., Huang, S. M. (2012). Chapter 15 - Drug Interactions. Principles of Clinical Pharmacology. Academic Press, 239-257. doi: http://doi.org/10.1016/b978-0-12-385471-1.00015-5

4. Dobrova, A. O., Materiienko, A. S., Golovchenko, O. S., Georgiyants, V. A. (2017). The biopharmaceutical study of doxycycline interaction with mineral waters and soft drinks in vitro. Clinical Pharmacy, 21 (3), 55-62. doi: http://doi.org/10.24959/cphj.17.1434

5. Dobrova, A. O., Golovchenko, O. S., Georgiyants, V. A. (2021). Modelling and investigation of amoxicillin chemical interaction with mineral waters containing a significant amount of calcium and magnesium salts. Pharmacia, 68 (1), $101-107$. doi: http://doi.org/10.3897/pharmacia.68.e39573 
6. Lasia, A. (2014). Electrochemical Impedance Spectroscopy and its Applications. New York: Springer, 367. doi: http://doi.org/10.1007/978-1-4614-8933-7

7. Barsoukov, E., Macdonald, J. R. (2018) Impedance Spectroscopy: Theory, Experiment, and Applications. John Wiley \& Sons, Inc. doi: http://doi.org/10.1002/9781119381860

8. Kozheshkurt, V., Antonenko, Ye., Shtoda, D., Slipchenko, O., Katrych, V. (2018). Possibilities of Impedance Spectroscopy for the Study of Bioliquids 2018. 9th International Conference IEEE, 260-263 doi: http://doi.org/10.1109/uwbusis.2018.8520236

9. Selwin Joseyphus, R., Viswanathan, E., Justin Dhanaraj, C., Joseph, J. (2012). Dielectric properties and conductivity studies of some tetradentate cobalt(II), nickel(II), and copper(II) Schiff base complexes. Journal of King Saud University - Science, 24 (3), 233-236. doi: http://doi.org/10.1016/j.jksus.2011.03.004

10. Khan, F. (2007). Electrochemical study and dependence of 'transition state' in Co(II) and Ni(II) complexes with some antibiotics and cephalothin. Eclética Química, 32 (3), 73-83. doi: http://doi.org/10.1590/s0100-46702007000300010

11. Jenkins, A. J., Valentine, J. L.; Mozayani, A., Raymon, L. (Eds.) (2012). Antimicrobial Drugs. Handbook of Drug Interactions. Humana Press, 385-411. doi: http://doi.org/10.1007/978-1-61779-222-9_10

12. Lambs, L., Brion, M., Berthon, G. (1984). Metal ion-tetracycline interactions in biological fluids. Part 3. Formation of mixedmetal ternary complexes of tetracycline, oxytetracycline, doxycycline and minocycline with calcium and magnesium, and their involvement in the bioavailability of these antibiotics in blood plasma. Agents and Actions, 14 (5-6), 743-750. doi: http://doi.org/10.1007/bf01978919

13. Guerra, W., Silva, I. R., Azevedo, E. A., Monteiro, A. R. de S., Bucciarelli-Rodriguez, M., Chartone-Souza, E. et. al. (2006). Three new complexes of platinum(II) with doxycycline, oxytetracycline and chlortetracycline and their antimicrobial activity. Journal of the Brazilian Chemical Society, 17 (8), 1627-1633. doi: http://doi.org/10.1590/s0103-50532006000800021

14. State Pharmacopoeia of Ukraine (2015). Kharkiv: State Enterprise: "Scientific-and-expert Pharmacopeial Centre", 1128.

15. Bard, A. J., Faulkner, L.R. (2001). Electrochemical methods: fundamentals and applications. John Wiley \& Sons, Inc., 864.

16. Tao, Z., Liu, G., Li, Y., Zhang, R., Su, H., Li, S. (2020). Electrochemical Investigation of Tetrazolium Violet as a Novel Copper Corrosion Inhibitor in an Acid Environment. ACS Omega, 5 (9), 4415-4423. doi: http://doi.org/10.1021/acsomega.9b03475

17. Christy, F. A., Shrivastav, P. S. (2011). Conductometric Studies on Cation-Crown Ether Complexes: A Review. Critical Reviews in Analytical Chemistry, 41 (3), 236-269. doi: http://doi.org/10.1080/10408347.2011.589284

18. Khammas, Z. A. A., Rashid, R. A. (2016). Visible spectrophotometric analysis for the mutual determination of doxycycline hydrochloride and iron in real samples after cloud point extraction. International Journal of Chemical Science, 14 (2), 955-977.

19. Javed, J., Zahir, E. (2017). Thermodynamic studies of iron chelation with doxycycline in acidic medium. Russian Journal of Physical Chemistry A, 91 (6), 1045-1050. doi: http://doi.org/10.1134/s0036024417060115

Received date 18.01.2021

Accepted date 23.02.2021

Published date 28.02.2021

Anna Dobrova, Postgraduate Student, Department of Pharmaceutical Chemistry, National University of Pharmacy, Pushkinska str., 53, Kharkiv, Ukraine, 61002

E-mail: anna.dobrova08@gmail.com

Yevhenii Antonenko, Researcher, Senior lecturer, Department of Physical and Biomedical Electronics and Complex Information Technologies, V. N. Karazin Kharkiv National University, Svobody sq., 4, Kharkiv, Ukraine, 61022

E-mail: antonenko@karazin.ua

Olga Golovchenko, PhD, Associate Professor, Department of Pharmaceutical Chemistry, National University of Pharmacy, Pushkinska str., 53, Kharkiv, Ukraine, 61002

E-mail: golyas26@ukr.net

Natalia Harna, PhD, Associate Professor, Department of Pharmaceutical Chemistry, National University of Pharmacy, Pushkinska str., 53, Kharkiv, Ukraine, 61002

E-mail: garnayan@ukr.net

Svitlana Garna, Doctor of Pharmaceutical Sciences, Professor, Department of Quality, Standardization and Certification of Medicines, Institute for Improvement of Qualification of Pharmacists, National University of Pharmacy, Pushkinska str., 53, Kharkiv, Ukraine, 61002

E-mail: kssi-ipksf@nuph.edu.ua

Victoriya Georgiyants, Doctor of Pharmaceutical Sciences, Professor, Head of Department, Department of Pharmaceutical Chemistry, National University of Pharmacy, Pushkinska str., 53, Kharkiv, Ukraine, 61002

E-mail: vgeor@ukr.net 